\title{
The effective conductivity of strongly nonlinear media: the dilute limit
}

\author{
François Willot ${ }^{\mathrm{a}, \mathrm{b}}$ \\ ${ }^{a}$ Mines ParisTech, PSL - Research University, Centre for Mathematical Morphology, \\ 35 rue Saint-Honoré, F-77300 FONTAINEBLEAU, France. \\ ${ }^{b}$ Mines ParisTech, PSL - Research University, Centre for Materials, \\ 63-65 Rue Henri Auguste Desbruères, F-91100 CORBEIL-ESSONNES, France.
}

Published in: International Journal of Solids and Structures 184 (2020) pp. 287-295 [Special issue on "Physics and Mechanics of Random Structures: From Morphology to Material Properties"]. DOI: 10.1016 /j.ijsolstr.2019.06.006.

\begin{abstract}
This work is a combined numerical and analytical investigation of the effective conductivity of strongly nonlinear media in two dimensions. The nonlinear behavior is characterized by a threshold value for the maximal absolute current. Our main focus is on random media containing an infinitesimal proportion $f \ll 1$ of insulating phase. We first consider a random conducting network on a square grid and establish a relationship between the length of minimal paths spanning the network and the network's effective response. In the dilute limit $f \ll 1$, the network's effective conductivity scales, to leading-order correction in $f$, as $\sim f^{\nu}$ with $\nu=1$ or $\nu=1 / 2$, depending on the direction of the applied field with respect to the grid. Second, we introduce coupling between local bonds, and observe an exponent $\nu \approx 2 / 3$. To interpret this result, we derive an upper-bound for the length of geodesics spanning random media in the continuum, relevant to media with a dilute concentration of heterogeneities. We argue that $\nu=2 / 3$ for random composites in the continuum with homogeneously-distributed, monodisperse particles, in two dimensions.
\end{abstract}

Keywords: Minimal paths; Homogenization; Composites; Conductivity; Probabilistic models

\section{Introduction}

A broad range of physical phenomena in material science involve the emergence of surfaces or paths of minimal energy. Examples include domain walls in random Ising systems (Huse and Henley, 1985), current localization in varistors (or non-Newtonian fluid flow) (Donev et al., 2002), the onset of voltage in polycrystals (Haslinger and Joynt,

Email address: francois.willot@ensmp.fr (François Willot) 
2000), or ductile fracture (De Arcangelis et al., 1989; Bouchaud et al., 1993). It is common to address these problems using idealized random network models (see Donev et al. (2002); Duxbury et al. (2006) and references therein). In highly-nonlinear networks, which are relevant to varistors and superconductors, localized flow paths emerge at special points, which act as thresholds for the network's macroscopic response (Donev et al., 2002). These paths are the solutions of the "shortest-path" and "minimum-cut" problems (Duxbury et al., 2006). The equivalence between a network's effective response and an optimization problem on graphs is demonstrated by the min-cut/max-flow theorem (Elias et al., 1956). Interestingly, the optimal surfaces exhibit, in the presence of disorder, rich self-affine structures. Their roughness, notably, presents non-standard scaling laws (Bovier et al., 1986; Alava and Duxbury, 1996).

In a numerical study of an "analog electric network" that mimic perfect-plasticity, it is argued that minimal surface problems are related to the effective yield stress of disordered media (Roux and Hansen, 1992). Optimal surfaces are obtained by minimizing the sum of the capacity (analog to the yield stress) taken over each bond that crosses the surface. The behavior of such minimal surface area with respect to the spatial distribution of heterogeneities, remains, however, an open problem.

This is especially true when considering a continuum medium, where the heterogeneities are characterized by the inclusions shape and their spatial distribution. Consider as a model problem a periodic porous medium in the continuum, in 2D. Using limit analysis, Drucker (1966) derived bounds on the effective yield stress $y_{0}$ of periodic porous media under plane strain. The pores are embedded in a rigid, ideally-plastic matrix with Tresca or Von Mises yield criterion $y$. Drucker's bounds demonstrate that the leading-order term to the effective flow stress scales as $\sim f^{1 / 2}$ in the limit of vanishingly small porosity $f$, i.e.:

$$
y_{0}=y\left[1-a f^{1 / 2}+o\left(f^{1 / 2}\right)\right], \quad f \rightarrow 0,
$$

with prefactor $a$ depending on the geometry of the inclusions. The infinite slope of the flow stress $\left(\partial y_{0} / \partial f=\infty\right)$ when $f=0$ underlines the strong effect of the voids in this limit. The trial displacement fields used to derive the upper-bound are piecewiseconstant and present surface discontinuities in the matrix (Francescato et al., 2004). Optimal upper-bounds are thus attained by minimal surfaces. In plane strain, these surfaces reduce to minimal paths, or in geometrical terms, geodesics. The paths are minimal in the sense that they have smallest cut in the matrix and span the array of pores in a direction that depends on the applied macroscopic loading. Examples for a 2D square array of pores with prescribed "simple" and "pure shear" (45-rotated) loadings are given in (Idiart et al., 2009).

Theoretical results suggest that the effective yield stress of 2D random porous media also exhibits power-laws with non-integer exponents, in the dilute porosity limit $f \rightarrow 0$ (Sab, 1994). In a square lattice model made of broken bonds in proportion $f$, the exponent for the effective yield stress is found to be 1 for minimal paths oriented along the directions of the lattice, and 1/2 along the diagonal (Roux and François, 
1991). The "second-order" nonlinear homogenization theory predicts a fractional exponent $2 / 3$ in the dilute limit $f \rightarrow 0$, in the plane strain problem (Ponte Castañeda, 2002), which suggests a lower influence of the pores than in the periodic problem. In this work, we estimate the length of geodesics in random continuum models made of a dilute concentration of particles. The scaling laws obtained are compared to the effective conductivity of various resistor networks, computed numerically.

This article is articulated around two main sections. In the first one (Section 2) we consider a random resistor network on a square grid, in two dimensions, and establish scaling laws for the effective conductivity with respect to $f$, the proportion of broken bonds, in the limiting case $f \rightarrow 0$. In the second one (Section 3), we derive an upperbound on the length of geodesics for a Boolean model of disks in the continuum, and interpret the scaling laws for the effective conductivity obtained in Section 2. We conclude in Section 4.

\section{Nonlinear resistor network: the dilute limit}

\subsection{Resistor network}

Consider a resistor network on a square grid (Fig. 1b) with nodes $\boldsymbol{x}=\left(x_{1}, x_{2}\right) \in \mathbb{Z}^{2}$. Each node is linked to its four closest neighbours by bonds oriented along $\mathbf{e}_{1}$ and $\mathbf{e}_{2}$ (see Fig. 1a). At each node $\boldsymbol{x}$ lies a potential $\phi(\boldsymbol{x})$, and along each bond $\left(\boldsymbol{x}, \boldsymbol{x}+\mathbf{e}_{i}\right)$ lies component $J_{i}(\boldsymbol{x})$ of the current and component $E_{i}(\boldsymbol{x})$ of the electric field. Following Kirchhoff's law:

$$
\sum_{i=1}^{2}\left[J_{i}(\boldsymbol{x})-J_{i}\left(\boldsymbol{x}-\mathbf{e}_{i}\right)\right]=0, \quad E_{i}(\boldsymbol{x})=-\left[\phi\left(\boldsymbol{x}+\mathbf{e}_{i}\right)-\phi(\boldsymbol{x})\right],
$$

for all $\boldsymbol{x}$ and $i=1,2$. Each bond in the resistor network is either insulating, in which case $J_{i}(\boldsymbol{x})=0$, or conducting. The conducting bonds follow the nonlinear constitutive law:

$$
J_{i}(\boldsymbol{x})=\left\{\begin{array}{cl}
\sigma E_{i}(\boldsymbol{x}) & \text { if } \sigma\left|E_{i}\right| \leq J_{0} \\
J_{0} \operatorname{sign}\left(E_{i}(\boldsymbol{x})\right) & \text { if } \sigma\left|E_{i}\right|>J_{0}
\end{array}\right.
$$

where $\operatorname{sign}(\bullet)=\bullet /|\bullet|$ is the $\operatorname{sign}$ function, $J_{0}>0$ is the yield current and $\sigma>0$ is a parameter used for numerical regularization (see Fig. 1c). In the limit $\sigma \rightarrow \infty$, Eq. (3) reads:

$$
\begin{aligned}
& J_{i}=J_{0} \quad \text { if } \quad E_{i}>0, \\
& J_{i}=-J_{0} \quad \text { if } \quad E_{i}<0 \text {, } \\
& \left|J_{i}\right|<J_{0} \quad \text { if } \quad E_{i}=0 \text {. }
\end{aligned}
$$

This constitutive law is obtained as the limiting behavior of "power-law materials". This class of materials, extensively studied in the context of nonlinear homogenization theories (Ponte Castañeda and Suquet, 1997), is characterized by local potentials which are power-law of the electric or current fields. In the present case, refered to as "strongly 


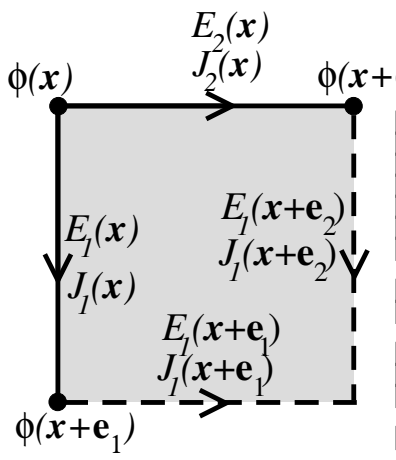

(a)

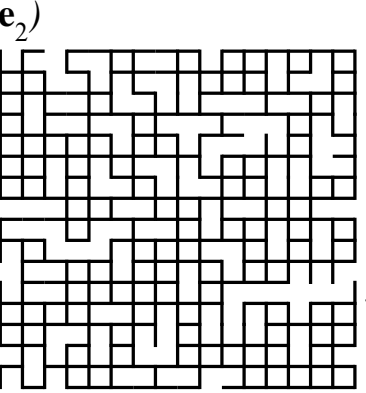

(b)

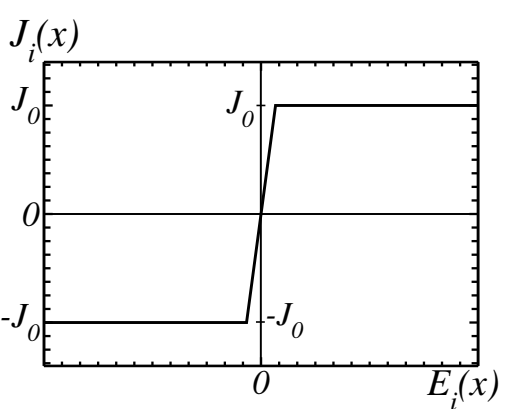

(c)

Figure 1: (a) Two bonds originating from a node at point $\boldsymbol{x}$ and joining nodes at $\boldsymbol{x}+\mathrm{e}_{1,2}$ (solid lines); other nearby bonds are represented as dotted lines. (b) $16 \times 16$-nodes random conducting network. Conducting bonds shown in black, insulating bonds omitted. (c) Nonlinear constitutive law (3) satisfied along each conducting bond.

nonlinear", the potential corresponding to the constitutive law (4) is not strictly convex, and fields may localize along critical paths (Duxbury et al., 2006).

For numerical purposes, we assume that the medium is periodic in the two directions and is given by its elementary cell $\Omega=[0 ; M-1]^{2}$ made of $M^{2}$ bonds. A random configuration of $\Omega$ is depicted in Fig. (1b) for $M=16$. In domain $\Omega$, the network is made up of two phases, the phase label following a Bernouilli distribution, that is, a bond has constitutive law (3) with probability $1-f$ and is insulating with probability $f$. The vector fields $\boldsymbol{E}$ and $\boldsymbol{J}$ are $\Omega$-periodic whereas the potential is the sum of a periodic and linear function:

$$
\phi(\boldsymbol{x})=-\overline{\boldsymbol{E}} \cdot \boldsymbol{x}+\phi^{*}(\boldsymbol{x}), \quad \phi^{*} \#,
$$

where $\overline{\boldsymbol{E}}$ is a remote field applied at infinity and \# denotes doubly-periodic fields. Problem (2)-(3)-(5) is solved exactly on the finite domain $\Omega$ using the Fourier-based method with "discrete" Green operator appropriate to resistor networks (Willot et al., 2014). As for the iterative algorithm, we make use of the so-called "augmented Lagrangian" scheme (Michel et al., 2001). When applied to materials with nonlinear threshold law (3), this scheme requires one to solve the equation in $\boldsymbol{E}$ :

$$
\sigma_{0} \boldsymbol{E}+\boldsymbol{J}(\boldsymbol{E})=\boldsymbol{P}
$$

for arbitrary $\boldsymbol{P}$. Making use of (3), the solution to this problem is straightforward. In the above, $\sigma_{0}$ is the reference conductivity of the homogeneous medium, which we choose equal to 1 . We also choose $J_{0}=1$ and restrict ourselves to $|\overline{\boldsymbol{E}}|=1$, so that the problem depends on $\sigma, f, M$ and the direction of the applied field $\overline{\boldsymbol{E}}$. Convergence is obtained when current conservation is observed at all nodes (Eq. 2). Our convergence criterion is the $L_{2}$-norm of the divergence of the current field:

$$
|\operatorname{div} \boldsymbol{J}|=\left(\sum_{\boldsymbol{x} \in \Omega} \sum_{i=1}^{2}\left|J_{i}(\boldsymbol{x})-J_{i}\left(\boldsymbol{x}-\mathbf{e}_{i}\right)\right|^{2}\right)^{1 / 2}<\eta
$$


with $\eta=10^{-10}$. Convergence is somehow erratic but nevertheless obtained (Fig. 2a).
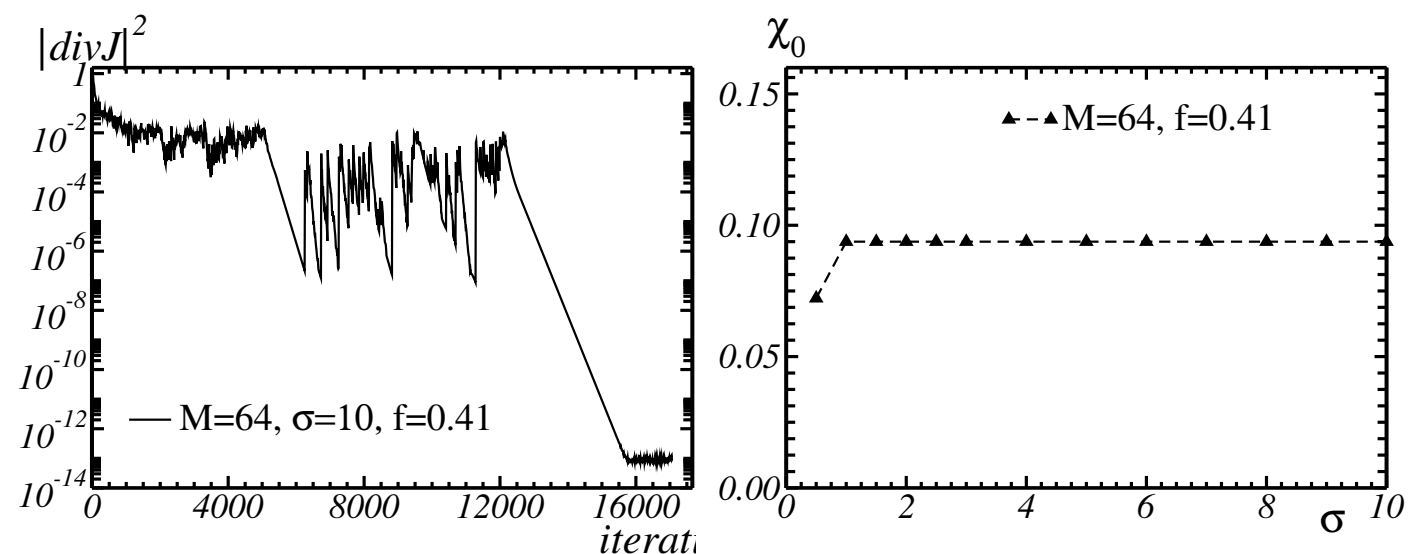

Figure 2: Random network in a $64 \times 64$ grid. (a) Convergence criterion (7) vs. number of iterations. (b) Effective conductivity $\chi_{0}$ vs. slope $\sigma$, with $\bar{E}=\mathbf{e}_{1}$.

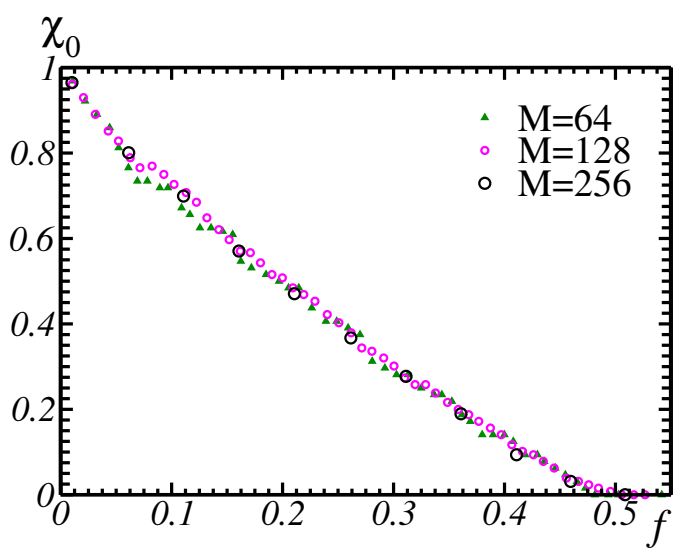

(a)

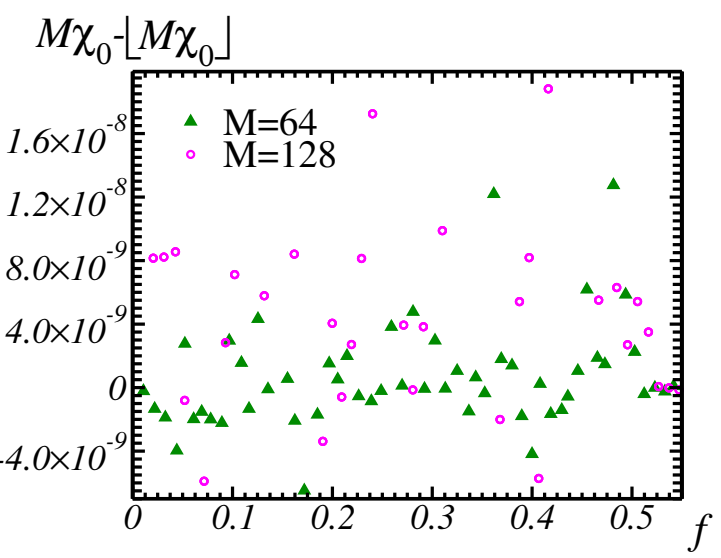

(b)

Figure 3: (a) Effective scalar conductivity $\chi_{0}$ vs. insulator fraction $f$, for various grid sizes, with applied electric $\overline{\boldsymbol{E}}=\mathbf{e}_{1}$. (b) Fractional part of $M \chi_{0}$ showing that $M \chi_{0}$ is an integer.

Define the effective conductivity tensor as:

$$
\overline{\boldsymbol{J}}=\langle\boldsymbol{J}(\boldsymbol{x})\rangle_{\Omega}=\overline{\boldsymbol{\chi}} \cdot \overline{\boldsymbol{E}},
$$

where $\langle\bullet\rangle_{\Omega}$ denotes a spatial mean over $\Omega$. We also define the scalar effective conductivity, that depends on the orientation of the applied field:

$$
\chi_{0}=\frac{\overline{\boldsymbol{J}} \cdot \overline{\boldsymbol{E}}}{|\overline{\boldsymbol{E}}|^{2}} .
$$


We seek for high values of the slope at origin $\sigma$ so that $\chi_{0}$ represents the effective yield current. In the rest of this work, we set $\sigma=10$, so that the effective behavior has reached its yield value when $\langle\boldsymbol{E}\rangle=\overline{\boldsymbol{E}}$ (Fig. 2 b, with $\boldsymbol{E}=\mathbf{e}_{1}$ ).

\subsection{FFT results}

Using $\sigma=10$, the effective conductivity is computed on grids of increasing size along each dimension $M=64,128$ and 256 and insulator fraction $f$ in the range $\left[0 ; f_{c}\right]$ where $f_{c}=1 / 2$ is the bond-percolation threshold for square lattices (Fig. 3a). We observe that the effective conductivity $\chi_{0}$ for all FFT data point on this graph is of the form $k / M$ where $0 \leq k \leq M$ is an integer, up to an error of less than $10^{-7}$ (Fig. $3 \mathrm{~b}$ ). A connection may be established between this property and the onset of a minimal path (of integer length) along which the electric field localizes. This is illustrated in Figs. (4) which shows component $E_{1}$ of the electric field, represented as an image (Fig. 4a), as well as the grid itself (Fig. 4b). On the dual graph, there exists two paths of minimal length (Fig. 4b) that span the medium in the direction $\mathbf{e}_{2}$ transverse to the applied field (horizontal on the image). The length of a path is defined by counting a unit cost for crossing a conducting bond and 0 for an insulating bond, thereby minimizing the number of conducting bonds crossed by the path. Using this definition, the two minimal paths shown in Fig. (4b) have normalized length $\xi=17 / M$ and we notice that $\chi_{0}=\xi J_{0}$. It is obvious that $\xi J_{0}$ is an upper-bound of $\chi_{0}$. Conversely, the existence of a divergence-free current field with mean $\xi J_{0}$ is a consequence of the "max-flow min-cut" theorem (Dantzig and Fulkerson, 2003). Hence, $\xi=\chi_{0} / J_{0}$.

The non-unicity of the electric field is a consequence, in general, of the non-unicity of minimal paths. Assume that there is a closed loop in the set of minimal paths on the dual graph such as, for instance, two minimal paths joining at infinity. Along the bonds crossing the loop, the current field is $J_{0}$ and the electric field is larger than, or equal to, $J_{0} / \sigma$. Therefore, if the electric component along any of these bonds is larger than $J_{0} / \sigma+\delta E$ with $\delta E>0$, a solution of the problem may be defined by adding a quantity smaller than $\delta E$ to the potential $\phi(\boldsymbol{x})$ at each node $\boldsymbol{x}$ located in the interior of the loop. The electric fields $\boldsymbol{E}$ should be different in each solution, while the current field $\boldsymbol{J}$, and the overall energy $(1 / 2) \sum_{\Omega} \boldsymbol{E} \cdot \boldsymbol{J}$, is unique.

The length of minimal paths in random lattices is closely related to the "time constant" in first-passage percolation theory (Kesten, 1987). In this problem, domain that grows with respect to a discrete time $t$ is considered. Growth at each time step is controlled by the value along each bond at the frontier of the domain. After a long time $(t \rightarrow \infty)$, the region asymptotically defines a limiting shape (Hoffman, 2008) which in general, is not a ball (Durrett and Liggett, 1981), due to the anisotropy of the lattice. Thus, if $s\left(\boldsymbol{x}, \boldsymbol{x}^{\prime}\right)$ is the minimal length between two points $\boldsymbol{x}$ and $\boldsymbol{x}^{\prime}$, we expect $s\left(\boldsymbol{x}, \boldsymbol{x}^{\prime}\right) /\left|\boldsymbol{x}-\boldsymbol{x}^{\prime}\right|$ to converge to a finite value when $\left|\boldsymbol{x}-\boldsymbol{x}^{\prime}\right| \rightarrow \infty$, which depends on the direction of $\boldsymbol{x}-\boldsymbol{x}^{\prime}$. Equivalently, $\chi_{0}$ depends on the loading direction $\overline{\boldsymbol{E}}$ in the square lattice, as expected.

We now perform FFT computations of the effective conductivity $\chi_{0}$ for small values

of $f$, equal to $10^{-4}, 10^{-3}, 10^{-2}$ and $10^{-1}$ (Fig. 5). A fit of the data provides $\chi_{0} \approx 1-\sqrt{f}$, 


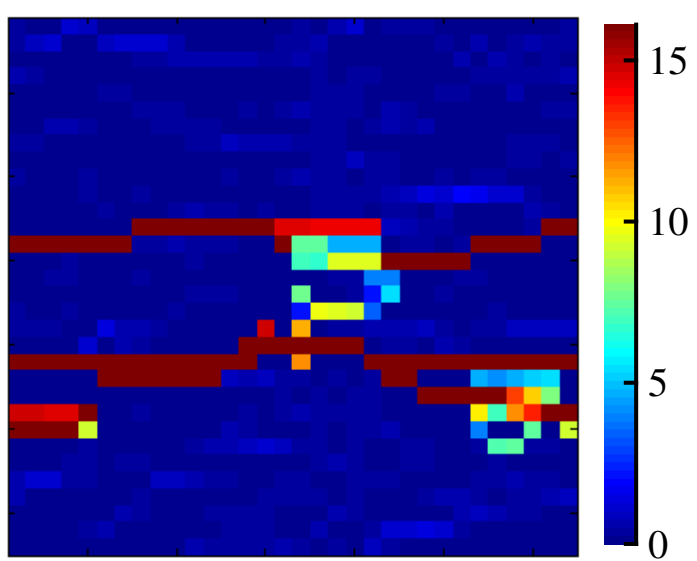

(a)

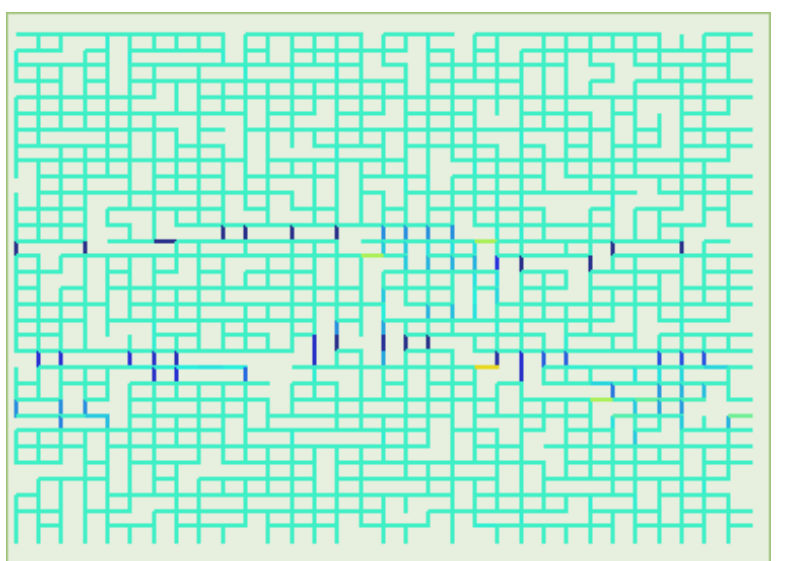

(b)

Figure 4: $\quad$ FFT results obtained on a grid $\Omega$ of $32 \times 32$ nodes, with $f=0.2$ and $\overline{\boldsymbol{E}}=\mathbf{e}_{1}$ where $\mathbf{e}_{1}$ is oriented top to bottom. (a) Component $E_{1}$ of the electric field, each bond being represented by a voxel. (b) Square grid. Missing bonds are insulating, other bonds are colored according to the value of the electric field: highest values in dark blue, lowest in green-blue, and intermediate values in yellow.

with nearly unit prefactor, when $\overline{\boldsymbol{E}}$ is oriented along direction $\mathbf{e}_{1}+\mathbf{e}_{2}$ (red symbols). FFT results obtained for a macroscopic field oriented along $\mathbf{e}_{1}$, are less conclusive (black symbols) but point nevertheless to a linear correction $\chi_{0} \approx 1-2.7 f$.

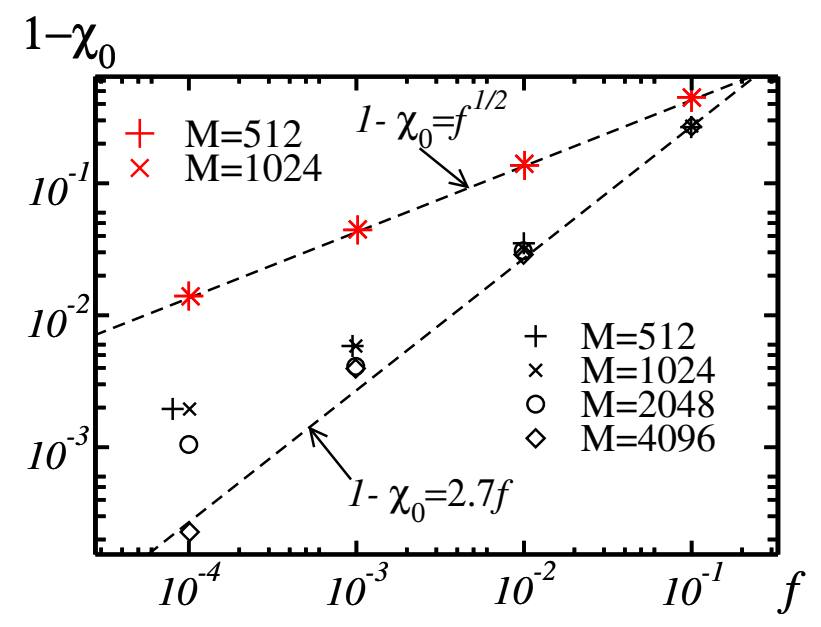

Figure 5: FFT results for the effective conductivity in the random resistor network. Red symbols: loading in the (1,1) direction $\overline{\boldsymbol{E}}=\mathbf{e}_{1}+\mathbf{e}_{2}$. Black symbols: loading in the (1,0) direction $\overline{\boldsymbol{E}}=\mathbf{e}_{1}$.

As pointed out by Roux and François (1991), the scaling law correction $\sim f^{1 / 2}$ in the $(1,1)$ direction is related to the rugosity (width in the transverse direction) of minimal paths. Suppose that a path of minimal length passes through two points $\boldsymbol{x}$ and $\boldsymbol{x}^{\prime}$ far from each other with $\boldsymbol{x}-\boldsymbol{x}^{\prime}$ parallel to $\mathbf{e}_{1}+\mathbf{e}_{2}$ (Fig. 6). In the absence of broken bonds, the length of the red and blue paths are the same. Therefore, if any broken bond appears inside the square $\left[x_{1} ; x_{1}^{\prime}\right] \times\left[x_{2} ; x_{2}^{\prime}\right]$ the length of the path will be reduced by 
1. Accordingly, the length of the minimal path normalized by $\left|\boldsymbol{x}^{\prime}-\boldsymbol{x}\right|$ will be reduced by $1 /\left(\sqrt{2}\left|x_{1}^{\prime}-x_{1}\right|\right)$ whereas $f$ increases by about $1 / L^{2}$, thus the normalized length of the minimal path change by a factor $\sim \sqrt{f}$. In the $(1,0)$ direction, minimal paths are mostly flat (Derrida and Vannimenus, 1983; Roux and François, 1991), therefore, the leading-order correction to $\chi_{0}$ is expected to be linear $(\sim f)$, as would be obtained for a straight line.

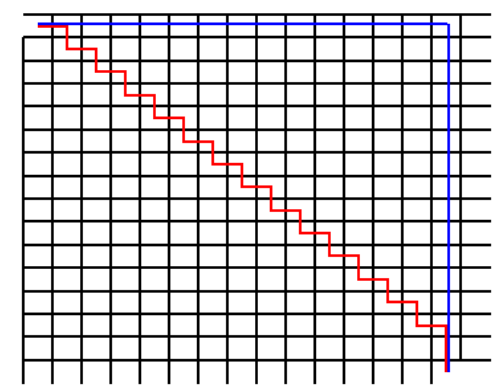

Figure 6: Two minimal paths (red and blue) joining two broken bonds (top-left and bottom-right) in the square lattice.

\subsection{Resistor network with local coupling}

Hereafter we consider a resistor network that may be used as a model for a material in the continuum. The elementary cell $\Omega$ is now made of a set of $M \times M$ pixels, rather than nodes. The electric and current field $\boldsymbol{E}(\boldsymbol{x})$ and $\boldsymbol{J}(\boldsymbol{x})$, and the potential field $\phi(\boldsymbol{x})$ are defined in each pixel. We keep the conservation and admissibility equations (2) and (5), i.e., we use finite differences, but consider instead of (3) the constitutive law:

$$
J_{i}(\boldsymbol{x})=\left\{\begin{array}{cc}
\sigma E_{i}(\boldsymbol{x}) & \text { if } \sigma|\boldsymbol{E}(\boldsymbol{x})| \leq J_{0}, \\
J_{0} E_{i}(\boldsymbol{x}) /|\boldsymbol{E}(\boldsymbol{x})| & \text { if } \sigma|\boldsymbol{E}(\boldsymbol{x})|>J_{0},
\end{array}\right.
$$

where $|\boldsymbol{E}(\boldsymbol{x})|^{2}=E_{1}(\boldsymbol{x})^{2}+E_{2}(\boldsymbol{x})^{2}$. Problem (2)-(5)-(10) is that of a random resistor network with local coupling between bonds pointing from the same node. As previously we solve this problem numerically using FFT computations, carried out using $\sigma=10$, this time on grids of $2048^{2}$ and $4096^{2}$ voxels. The augmented Lagrangian scheme is found to be somehow slow to deal with this problem, and we turn to the "accelerated scheme" (Eyre and Milton, 1999) with "discrete" Green operator (Willot et al., 2014). The accelerated scheme was originally devised for linear behavior. However, it is straightforward to extend it to nonlinear behavior, provided one is able to invert an equation of the type (6). We choose $\sigma_{0}=0.01$ for the conductivity of the reference medium. The effective conductivity $\chi_{0}$ is again defined by (9).

We consider the limiting case of a dilute concentration of insulators. FFT results for the effective behavior are represented in Fig. (7) as a function of the concentration of insulators, in log-log plot. The leading-order term in $f$ exhibits a power-law scaling with exponent $\approx 2 / 3$. We interpret this result in the following section. 


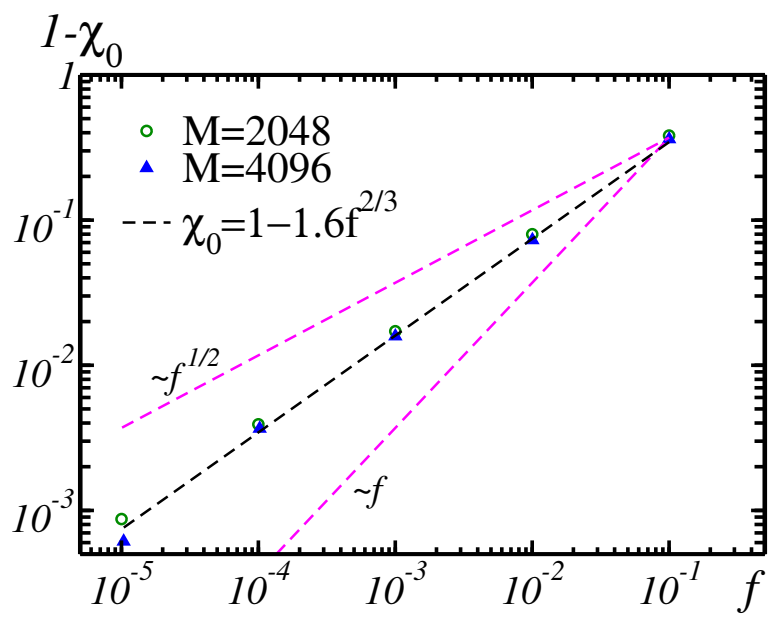

Figure 7: Log-log plot of $1-\chi_{0}$ vs. fraction of insulating bonds $f$, in the dilute limit $f \rightarrow 0$. Data points: FFT results. Black dotted line: power-law fit with $2 / 3$ exponent of FFT data points. Other dotted lines: power-law $f$ and $f^{1 / 2}$.

\section{Length of geodesics in $2 \mathrm{D}$ media}

In this section, we detail how to construct an upper-bound for the length of minimal paths in continuum media, restricted to particulate materials with dilute concentration of heterogeneities. The method has already been introduced by Willot (2015). For the sake of clarity, we derive it again in a simple way, in the case of a Boolean model of disks. We compare the bound with numerical results for the length of geodesics in finite-size systems. We also study in more details the rugosity exponent of the path used to construct the bound. Finally, we consider as an application binary mixtures where each phase is characterized by a nonlinear potential with non-zero yield current. The bound on geodesic is used to obtain estimates for the effective conductivity of such materials.

\subsection{Boolean model of disks}

Hereafter, a Boolean set (Matheron, 1975; Serra, 1981) of disks in $\mathbb{R}^{2}$, of surface fraction $0 \leq f \leq 1$ is considered. The disks have constant diameter $D>0$ and may interpenetrate. Their centers follow a homogeneous Poisson point process. Disks are crossed at no cost whereas the embedding medium is crossed at a unit cost. The distance between two points $\boldsymbol{A}$ and $\boldsymbol{B}$ therefore reads:

$$
\begin{aligned}
d(\boldsymbol{A}, \boldsymbol{B})= & \inf _{\boldsymbol{p} \in \mathcal{K}} \int_{0}^{1} \mathrm{~d} t \chi(\boldsymbol{p}(t))\left\|\partial_{t} \boldsymbol{p}(t)\right\|, \\
& \chi(\boldsymbol{M})=\left\{\begin{array}{cc}
0 & \text { if } \boldsymbol{M} \text { lies inside a disk, } \\
1 & \text { otherwise, }
\end{array}\right.
\end{aligned}
$$


where $\chi$ is the indicator function of the embedding medium, $\|\cdot\|$ is the Euclidean norm and:

$$
\mathcal{K}=\left\{\boldsymbol{p} \in \mathcal{C}\left([0 ; 1], \mathbb{R}^{2}\right), \quad \boldsymbol{p}(0)=\boldsymbol{A}, \quad \boldsymbol{p}(1)=\boldsymbol{B}\right\}
$$

is the set of continuous curves from $\boldsymbol{A}$ to $\boldsymbol{B}$. Some immediate properties of the function $d(\cdot, \cdot)$ follow from $(11)$. For all points $\boldsymbol{A}, \boldsymbol{B}$ and $\boldsymbol{C}$ :

$$
\begin{aligned}
d(\boldsymbol{A}, \boldsymbol{A}) & =0, \quad d(\boldsymbol{A}, \boldsymbol{B})=d(\boldsymbol{B}, \boldsymbol{A}) \geq 0, \\
d(\boldsymbol{A}, \boldsymbol{B}) & \leq d(\boldsymbol{A}, \boldsymbol{C})+d(\boldsymbol{B}, \boldsymbol{C}), \quad d(\boldsymbol{A}, \boldsymbol{B}) \leq\|\boldsymbol{B}-\boldsymbol{A}\|,
\end{aligned}
$$

so that $d$ is a pseudo-distance. Also from (11), it is clear that any continuous portion $\boldsymbol{p}^{\prime}$ of the path $\boldsymbol{p}$ is a minimal path between its extremal points. Accordingly, if the path $\boldsymbol{p}^{\prime}$ lies entirely in the embedding medium $\left(\chi\left(\boldsymbol{p}^{\prime}(t)\right) \equiv 1\right)$, it is necessarily straight. Therefore, minimal paths are unions of segments joining disk centers. Assume, for convenience, that the end points of the line segments are $\left(\boldsymbol{A} ; \boldsymbol{C}^{1} ; \ldots ; \boldsymbol{C}^{N} ; \boldsymbol{B}\right)(N \geq 0)$ where the $\boldsymbol{C}^{i}$ are disk centers of coordinates $\left(C_{1}^{i} ; C_{2}^{i}\right)$.

We now focus on the limiting behavior of the normalized distance:

$$
\xi=\frac{d(\boldsymbol{A}, \boldsymbol{B})}{L}, \quad L=\|\boldsymbol{A}-\boldsymbol{B}\| \rightarrow \infty
$$

Without loss of generality, we assume that $\boldsymbol{A}$ is the center of a disk at the origin of a Cartesian coordinate system $\left(\mathbf{e}_{1} ; \mathbf{e}_{2}\right)$ and that the line joining $\boldsymbol{A}$ and $\boldsymbol{B}$ is parallel to $\mathbf{e}_{1}$. When $\boldsymbol{A}$ is fixed and $L \rightarrow \infty$, the computation of $\xi$ amounts to study the limit shape of the set:

$$
S_{t}=\left\{\frac{1}{t} \boldsymbol{B} ; \quad d(\boldsymbol{A}, \boldsymbol{B}) \leq t\right\}, \quad \frac{1}{t} \boldsymbol{B}=\left(\frac{B_{1}}{t} ; \frac{B_{2}}{t}\right),
$$

as $t \rightarrow \infty$. The above can be regarded as a growth process in the continuum (Howard and Newman, 1997; Deijfen, 2003). In the present work, an isotropic Boolean set of disks is considered, and so the limiting shape $S_{\infty}$ of $S_{t}$ is a disk with radius $\lim _{L \rightarrow \infty}(1 / \xi)$.

In the rest of this work, we study the behavior of $\lim _{L \rightarrow \infty} \xi$ in the dilute limit $f \rightarrow 0$, and more exactly its leading-order correction in $f$. In effect, this amount to take the double limit:

$$
\lim _{f \rightarrow 0} \lim _{L \rightarrow \infty} \xi
$$

The two limits are not interchangeable, for if $\boldsymbol{A}$ and $\boldsymbol{B}$ are fixed and $f \rightarrow 0$, the minimal path between $\boldsymbol{A}$ and $\boldsymbol{B}$ is almost surely a straight line and $\xi=1$. Therefore, in the rest of this work $f \ll 1$ is fixed and $L \gg D$ is chosen sufficiently large that the minimal path between $\boldsymbol{A}$ and $\boldsymbol{B}$ passes through a very large number of disks $(N \gg 1)$.

\subsection{Three discs}

Consider three non-intersecting discs, identified by their centers $\boldsymbol{U}, \boldsymbol{V}$ and $\boldsymbol{W}$. We denote by $\boldsymbol{V}^{\prime}$ the projection of $\boldsymbol{V}$ onto the line $(\boldsymbol{U} \boldsymbol{W})$ and set $\ell=\left\|\boldsymbol{U}-\boldsymbol{V}^{\prime}\right\|$, 


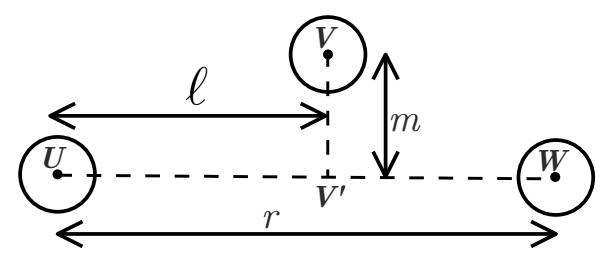

Figure 8: Three disks with centers $\boldsymbol{U}, \boldsymbol{V}$ and $\boldsymbol{W}$.

$m=\left\|\boldsymbol{V}-\boldsymbol{V}^{\prime}\right\|$ and $r=\|\boldsymbol{U}-\boldsymbol{W}\|$ (see Fig. 8). We are interested in the condition under which a path of minimal length joining $\boldsymbol{U}$ to $\boldsymbol{W}$ must pass through $\boldsymbol{V}$. Inequality:

$$
\|\boldsymbol{U}-\boldsymbol{V}\|-D+\|\boldsymbol{V}-\boldsymbol{W}\|-D \leq\|\boldsymbol{U}-\boldsymbol{W}\|-D
$$

yields:

$$
m \leq \frac{\sqrt{D(D+2 r)(D+2 r-2 \ell)(D+2 \ell)}}{2(D+r)} .
$$

In the dilute limit $f \rightarrow 0$, taking $r \gg D$ and $\ell \gg D$, (17) reduces to:

$$
m \leq \sqrt{2 D \ell(1-\ell / r)} .
$$

Assuming that $\boldsymbol{U}$ and $\boldsymbol{W}$ are fixed, equation (17) defines a domain of interest where disks may be looked for, in order to construct paths with small length. The surface of domain (18), scales as:

$$
S=2 \int_{0}^{r} \sqrt{2 D \ell(1-\ell / r)} \mathrm{d} \ell=\frac{\pi r \sqrt{D r}}{\sqrt{8}} .
$$

The width of this domain grows as $\sim \sqrt{r}$, which suggests a power-law behavior for the length of minimal paths in the dilute limit, in the continuum, following the argument of Roux and François (1991). This is detailed hereafter by the derivation of an upperbound on $\xi$.

\subsection{Upper-bound in the dilute limit}

We identify $\boldsymbol{U}$ with the center $\boldsymbol{C}^{i}$ of the $i^{\text {th }}$ disk on a path $\left(\boldsymbol{C}^{1} ; \ldots ; \boldsymbol{C}^{N}\right)$ and $\boldsymbol{W}$ as the "first" disk encountered in the direction $\mathbf{e}_{1}$ starting from $\boldsymbol{U}$, if the path was straight. The choice $\boldsymbol{C}^{i+1}=\boldsymbol{W}$ amounts to follow closely direction $\mathbf{e}_{1}$ and is not advantageous, unless there exists no disk $\boldsymbol{V}$ satisfying (17), an event that has a low probability. Hence, we consider instead the disk $\boldsymbol{V}$ satisfying (18) with minimal value of $\ell$. Clearly, $\ell \ll r$ and condition (18) becomes:

$$
m \leq \sqrt{2 D \ell} .
$$

The above property suggests the following iterative procedure for constructing a path $\left(\boldsymbol{C}^{0} ; \boldsymbol{C}^{1} ; \ldots ; \boldsymbol{C}^{N}\right)$ starting with the point $\boldsymbol{C}^{0}$. Knowing $\boldsymbol{C}^{i}, \boldsymbol{C}^{i+1}$ is the disk in the 


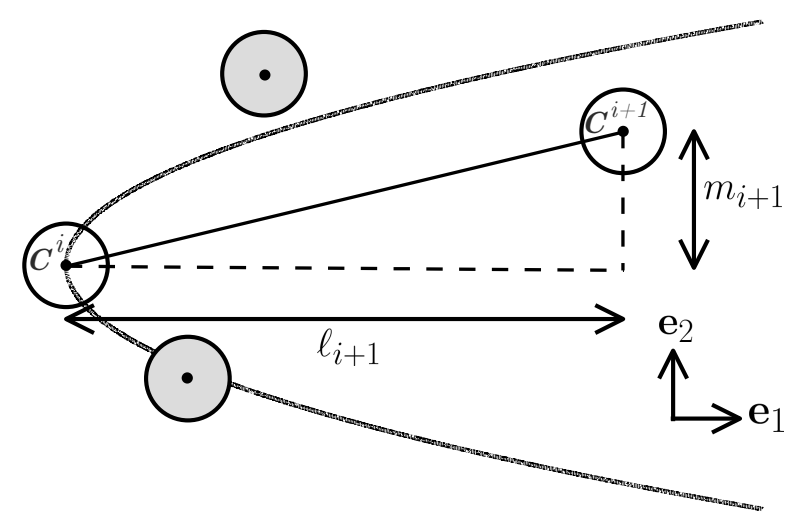

Figure 9: $\quad$ Method for choosing the disk $C^{i+1}$, knowing $C^{i}$.

domain delimited by two curves of equation $x_{2}=C_{2}^{i} \pm \sqrt{2 D\left|x_{1}-C_{1}^{i}\right|}$, with minimal coordinate $x_{1}$ along $\mathbf{e}_{1}$ (see Fig. 9):

$$
\begin{aligned}
\boldsymbol{C}^{i+1} & =\operatorname{arginf}_{\boldsymbol{C} \in \mathcal{K}^{\prime}} C_{1}, \\
\mathcal{K}^{\prime} & =\left\{\boldsymbol{C} \text { a disk center; } C_{1}>C_{1}^{i},\left|C_{2}-C_{2}^{i}\right| \leq \sqrt{2 D\left|C_{1}-C_{1}^{i}\right|}\right\} .
\end{aligned}
$$

This procedure may be compared to that used by Lee (1997) who derived a bound on the minimal length of self-avoiding paths in random lattices by selecting a series of points which move "as directly as possible" from one vertex to another. In the present method, however, we consider a continuum medium and the geodesics must follow a privileged direction.

Let us first replace condition (21) by:

$$
\begin{aligned}
\boldsymbol{C}^{i+1} & =\operatorname{arginf}_{\boldsymbol{C} \in \mathcal{K}^{\prime \prime}} C_{1} \\
\mathcal{K}^{\prime \prime} & =\left\{\boldsymbol{C} \text { a disk center; } C_{1}>C_{1}^{i}+D,\left|C_{2}-C_{2}^{i}\right| \leq \alpha \sqrt{D\left|C_{1}-C_{1}^{i}\right|}\right\}
\end{aligned}
$$

where $\alpha>0$ is a constant to be optimized on. We also request that $C_{1}^{i}>C_{1}+D$ so that the disks do not overlap. This technical assumption simplifies the analytical treatment,

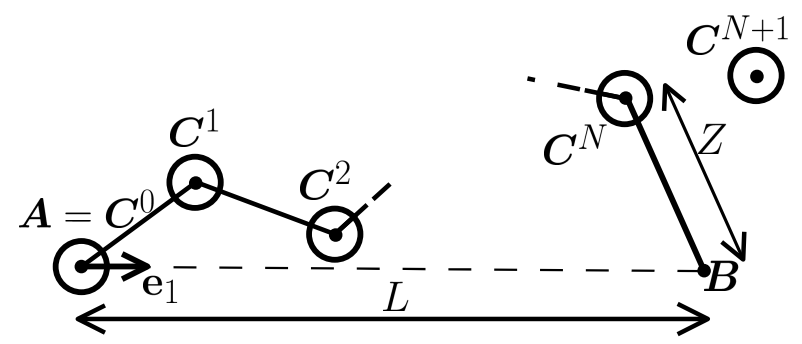

Figure 10: Path $\left(\boldsymbol{A} ; \boldsymbol{C}^{1} ; \ldots ; \boldsymbol{C}^{N} ; \boldsymbol{B}\right)$ defined by $(22)$ and used to deliver an upper-bound on the length of geodesics. 
and has no effect in the dilute limit where disks are "almost always" far from each other. Starting from $\boldsymbol{C}^{0}=\boldsymbol{A}$, we construct the path $\left(\boldsymbol{A} ; \boldsymbol{C}^{1} ; \ldots ; \boldsymbol{C}^{N} ; \boldsymbol{B}\right)$ where the number $N$ is chosen so that $\boldsymbol{C}^{N+1}$ is the first disk center with coordinate along $\mathbf{e}_{1}$ larger than $B_{1}$, i.e. $C_{1}^{N+1}>B_{1}$ and $C_{1}^{N} \leq B_{1}$. As in Section 3.2, we set $\ell_{i}=\left|C_{1}^{i}-C_{1}^{i-1}\right|, m_{i}=C_{2}^{i}-C_{2}^{i-1}$ $(i \geq 1)$. The path $(22)$ provides the following upper bound on $\xi=d(\boldsymbol{A}, \boldsymbol{B}) / L$ :

$$
\xi \leq \frac{\sum_{i=1}^{N}\left(\sqrt{\ell_{i}^{2}+m_{i}^{2}}-D\right)+Z}{\sum_{i=1}^{N} \ell_{i}}
$$

where $Z=\left\|\boldsymbol{C}^{N}-\boldsymbol{B}\right\|$ is the Euclidean distance from $\boldsymbol{C}^{N}$ to $\boldsymbol{B}$ (see Fig. 10). Rewrite (23) as:

$$
1-\xi \geq \frac{\sum_{i=1}^{N}\left(D+\ell_{i}-\sqrt{\ell_{i}^{2}+m_{i}^{2}}\right)-Z}{\sum_{i=1}^{N} \ell_{i}} \approx \frac{\sum_{i=1}^{N}\left[D-m_{i}^{2} /\left(2 \ell_{i}\right)\right]-Z}{\sum_{i=1}^{N} \ell_{i}} .
$$

To evaluate the above, we determine the mean of the $\ell_{i}$ and of $m_{i}^{2} / \ell_{i}$. The $m_{i}$ are uniform random variables in the interval $\left[-\alpha \sqrt{\ell_{i} D} ; \alpha \sqrt{\ell_{i} D}\right]$. The probability $P\left\{\ell_{i}>\ell\right\}$ is the probability that the domain delimited by the two curves in Fig. (9) and enclosed by the lines $x_{1}=C_{1}^{i-1}+D$ and $x_{1}=C_{1}^{i-1}+\ell_{i}$ contains no disk center. This probability is given by the Choquet capacity of a Poisson point process (Matheron, 1972) as $\exp (-\theta V)$ where $V$ is the size of the domain and $\theta=-4 \log (1-f) /\left(\pi D^{2}\right)$ is the intensity of the Poisson point process (i.e. the disks centers) used to build the Boolean set. We compute $V$ and obtain:

$$
P\left\{\ell_{i} \leq \ell\right\}=1-(1-f)^{16 \alpha /(3 \pi)\left[(\ell / D)^{3 / 2}-1\right]},
$$

as the cumulative probability function of the random variable $\ell_{i}$ in $[D ; \infty)$. The above yields, for the average of the $\ell_{i}$, using the variable change $\eta=(D / \ell)^{3 / 2}$ :

$$
\begin{aligned}
& \frac{1}{D N} \sum_{i=1}^{N} \ell_{i} \approx \frac{1}{D} \int_{\ell \geq D} \ell P\left\{\ell \leq \ell_{i} \leq \ell+\mathrm{d} \ell\right\} \\
& =1+\frac{2}{3}(1-f)^{-\frac{16 \alpha}{3 \pi}} E_{1 / 3}\left(\frac{-16 \alpha \log (1-f)}{3 \pi}\right)=\left(\frac{\pi}{4 \alpha \sqrt{6} f}\right)^{2 / 3} \Gamma\left(\frac{2}{3}\right)+o(1),
\end{aligned}
$$

where $E_{1 / 3}(t)=\int_{0}^{1} \mathrm{~d} \eta \mathrm{e}^{-t / \eta} \eta^{-5 / 3}$ is the exponential integral function of parameter $1 / 3$. Furthermore:

$$
\frac{1}{N} \sum_{i=1}^{N} \frac{m_{i}^{2}}{\ell_{i}} \approx \int_{\ell \geq D} \int_{m=0}^{\alpha \sqrt{\ell D}} \frac{m^{2}}{\ell} P\left\{\ell \leq \ell_{i} \leq \ell+\mathrm{d} \ell\right\} \frac{\mathrm{d} m}{\alpha \sqrt{\ell D}}=\frac{D \alpha^{2}}{3}
$$

At lowest order in $f$ :

$$
\xi \leq 1-\frac{2 \alpha^{2 / 3}\left(6-\alpha^{2}\right)}{3 \Gamma\left(\frac{5}{3}\right)}\left(\frac{2}{3 \pi}\right)^{2 / 3} f^{2 / 3}+O\left(f^{4 / 3}\right)+\frac{Z}{\sum_{i=1}^{N} \ell_{i}},
$$


where $\Gamma$ is the Gamma (or extended factorial) function. Furthermore, (26) entails $m_{i} \sim f^{-1 / 3}$ and:

$$
Z=\left|\sum_{i=1}^{N} m_{i}\right| \sim \sqrt{N} f^{-1 / 3} \sim \sqrt{L} .
$$

Accordingly the term $Z / \sum_{i=1}^{N} \ell_{i} \sim 1 / \sqrt{L}$ in (28) is negligible when $L$ is large. The choice $\alpha=\sqrt{3 / 2}$ in (28) then yields:

$$
\xi \leq 1-\frac{3}{\Gamma\left(\frac{2}{3}\right)}\left(\frac{3 f}{2 \pi}\right)^{2 / 3}+O\left(f^{4 / 3}\right) \approx 1-1.3534 f^{2 / 3} .
$$

We compare this dilute limit expansion to numerical results (see Appendix A for a description of our algorithm). The difference between the two paths is illustrated in Fig. (11) which shows the exact minimal path between two opposite corners of a square domain and the path predicted by bound (30) in a realization. Our numerical computations are carried out on random configurations containing on average 10,000 disks at increasing surface fractions $f=10^{-7}, \ldots, 10^{-1}$. For each value of $f, 20$ random configurations are averaged. We also compute numerically the bound (23). Results, indicated in Fig. (12), show an excellent agreement between bound (23) and expansion (30) (solid lines and black dots). A power-law fit on the numerical data for the exact minimal path length provides $\xi \sim 1-1.85 f^{0.67}$. This result, together with the asymptotic bound (30), suggests a scaling law with exponent exactly $2 / 3$ for the length of minimal path in the dilute regime $f \rightarrow 0$. It also provides a geometric interpretation of the exponent $\approx 2 / 3$ observed in Section 2.3 for the effective conductivity of the random resistor network with local coupling.

We close this section by a discussion of the rugosity of the minimal path and that of the path used to derive bound (30). The $m_{i}$ are independent variables with zero mean and finite variance:

$$
\left\langle m_{i}^{2}\right\rangle=\left(\frac{\pi}{12 f}\right)^{2 / 3} \Gamma\left(\frac{2}{3}\right) \frac{D^{2}}{2}=\left\langle\ell_{i}\right\rangle \frac{D}{2}=\frac{L D}{2 N},
$$

hence, by the Central Limit theorem, $(1 / N) \sum_{i} m_{i}$ is Gaussian for $N$ large. Accordingly, $Z$ follows the probability distribution function:

$$
P\{z<Z<z+\mathrm{d} z\} \frac{1}{\mathrm{~d} z}=\frac{2}{\sqrt{L D \pi}} \mathrm{e}^{-z^{2} /(L D)} .
$$

We have simulated the probability distribution function for $Z$ on 40,000 configurations each containing 15, 000 disks, with $f=0.01$ fixed. The value of $Z$ is estimated at various values of $L / D$ and compared with that obtained for the shortest path (Fig. 13). For the later, $Z$ is defined to be the deviation along the direction transverse to propagation, i.e. it is equal to $\left|x_{2}\right|$ where $\boldsymbol{x}$ lies on the shortest path such that $x_{1}=L$, the origin of the shortest path being 0 by convention. The data points for the numerical estimates 


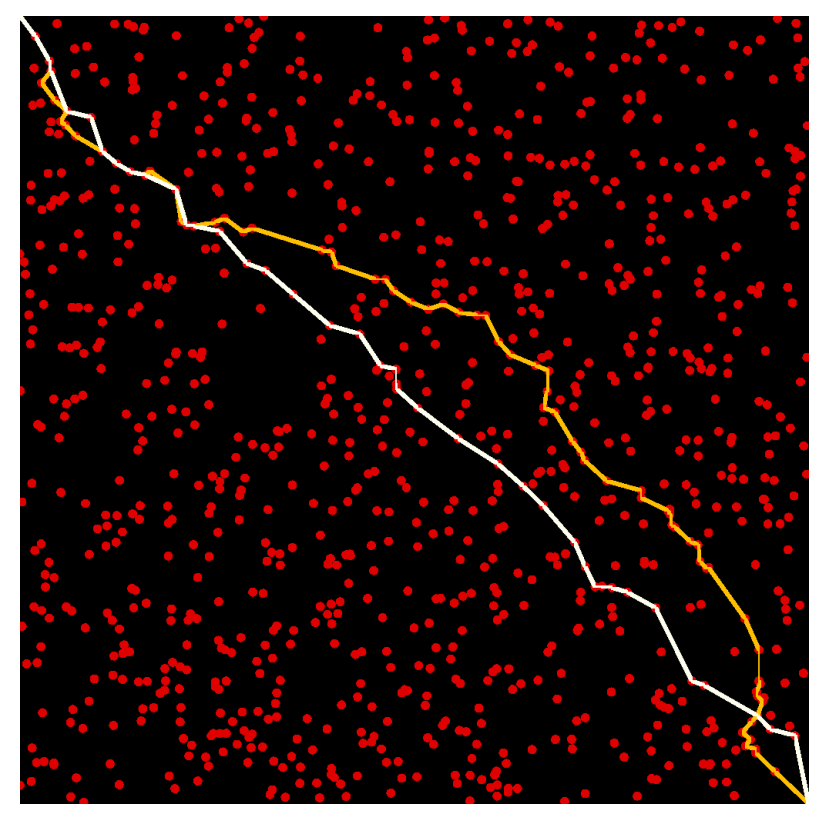

Figure 11: Boolean set of discs with surface fraction $f=10 \%$ and $10^{3}$ disks (in red). Yellow line: minimal path joining two opposite corners. White line: path used to derive bound (23).

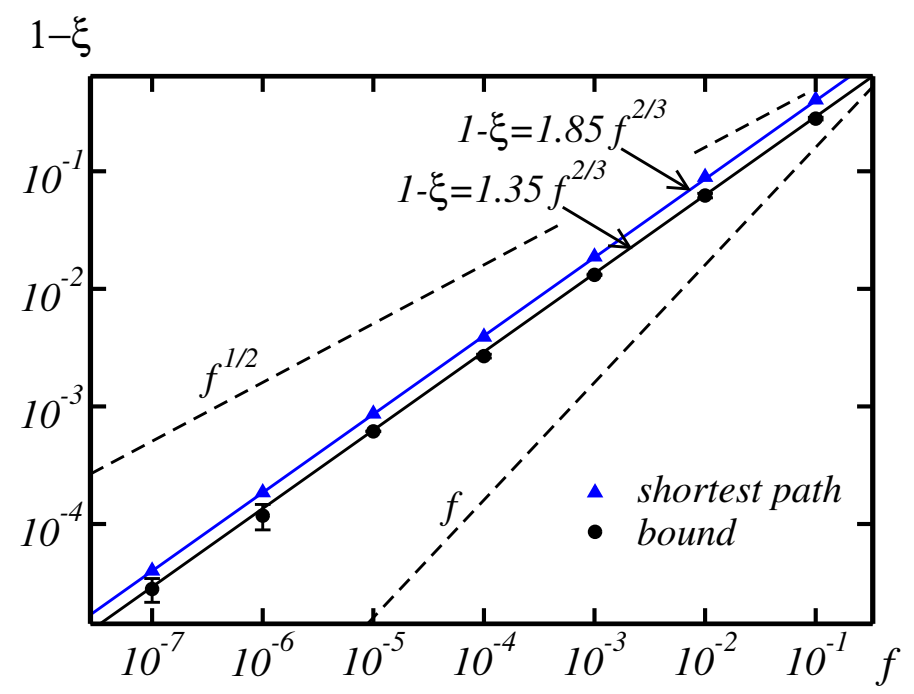

Figure 12: Leading-order correction to the length of geodesics $\xi$ vs. disk concentration $f$, in $\log$ - $\log$ plot. Black dots: numerical computation of bound (23); black solid line: analytical estimate (30); blue triangles: numerical computation of $\xi$ using the exact shortest path on finite-size systems; blue solid line: power-law fit of the latter; dotted lines: power-law corrections $\sim f$ and $\sim f^{1 / 2}$. 


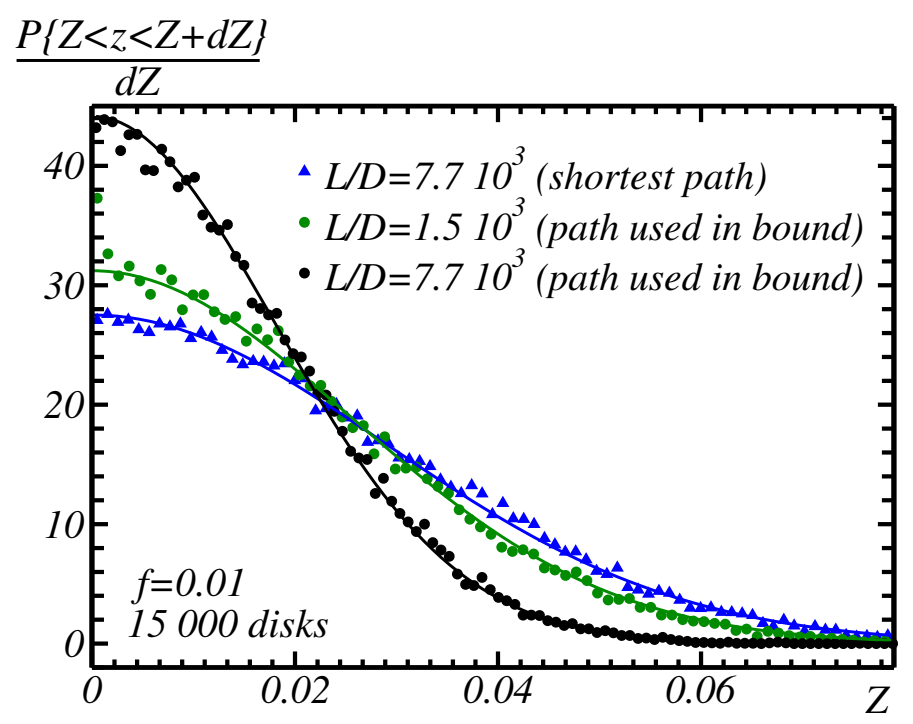

Figure 13: Probability distribution function for the random variable $Z$, representing the deviation transverse to the direction of propagation, computed numerically (symbols) and analytically (solid lines). Black and green data points: path used in bound (30). Blue data point: shortest path. Blue solid line: fit with a folded Gaussian distribution (32).

of $Z$ are in excellent agreement with (32). We also observe that the distribution of the variable $Z$ for the exact shortest path, at given value of $L / D$, is much wider than that of the bound (symbols in blue, Fig. 13). We study the width of the distribution of $Z$ hereafter.

Consider the path transverse fluctuations $w=\left(\left\langle Z^{2}\right\rangle-\langle Z\rangle^{2}\right)^{1 / 2}$ as a function of $L$, for fixed value of $f=10^{-2}, 10^{-3}$ and $10^{-4}$. It has been shown (Huse and Henley, 1985; Kardar and Zhang, 1987) that the minimal path is very rough with scaling law $w \sim L^{-2 / 3}$ as $L$ becomes large (see numerical computations, Fig. 14). As expected, this is not so for the path used to derive bounds (30) which exhibits a much more common scaling law $\sim L^{-1 / 2}$ (black line, Fig. 14). Thus, the exponent $2 / 3$ obtained in bound (30) is not directly linked to the "roughness exponent" related to the scaling law for $w$.

\subsection{Application: binary mixture with non-zero yield currents}

Assume that the constitutive law in each phase is of type (10) with yield current $J_{0}$ in the matrix and $J_{1}$ in inclusions, i.e.:

$$
\boldsymbol{J}=\min \left(\sigma|\boldsymbol{E}|, J_{c}\right) \frac{\boldsymbol{E}}{|\boldsymbol{E}|},
$$

with $J_{c}=1$ in the matrix and $J_{c}=J_{1}\left(0 \leq J_{1} \leq 1\right)$ in inclusions. According to the mincut, max-flow theorem, we now seek for a minimal path spanning the microstructure, with unit cost for the distance function in the matrix and cost $J_{1}$ in the inclusions. The same method as developed in Section 3.3 provides an upper-bound on the length of 


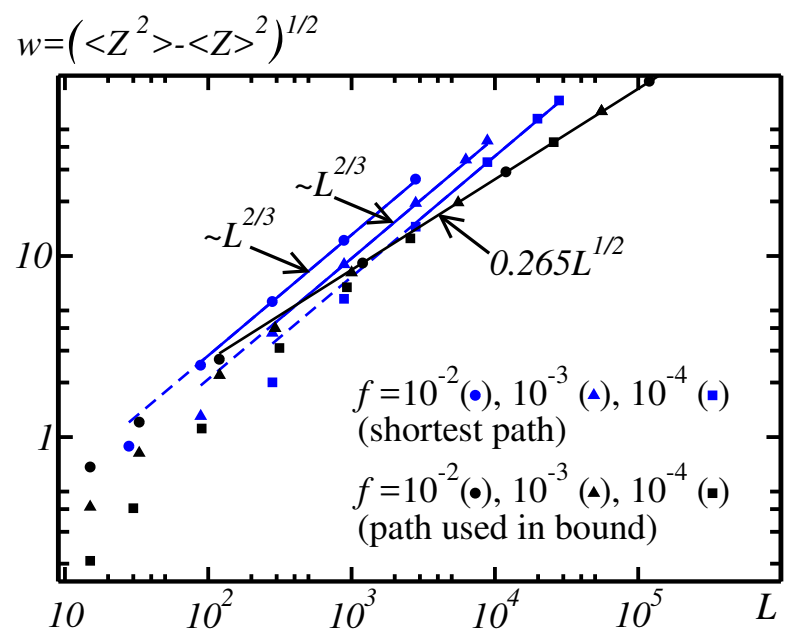

Figure 14: Transverse fluctuations $w$ as a function of the path length $L$ in log-log plot, for various values of $f$. Blue symbols: shortest path. Black symbols: path used in bound (30). Solid lines: power-law fit of the data.

geodesics of such materials (Willot, 2015). The path definition (22) remains unchanged, however (24) now becomes, after neglecting $Z$ :

$$
\xi \leq 1-\frac{\sum_{i=1}^{N}\left[\left(1-J_{1}\right) D-m_{i}^{2} /\left(2 \ell_{i}\right)\right]}{\sum_{i=1}^{N} \ell_{i}}
$$

Estimates for $\left\langle\ell_{i}\right\rangle$ and $\left\langle m_{i}^{2} / \ell_{i}\right\rangle$ (Eqs. 26, 27) remain unchanged and we obtain, after optimization on $\alpha$ :

$$
\xi \leq 1-\frac{3\left(1-J_{1}\right)^{4 / 3}}{\Gamma\left(\frac{2}{3}\right)}\left(\frac{3 f}{2 \pi}\right)^{2 / 3}+O\left(f^{4 / 3}\right) \approx 1-1.3534\left(1-J_{1}\right)^{4 / 3} f^{2 / 3},
$$

in the dilute limit $f \rightarrow 0$.

We carry out FFT computations of the effective conductivity of a random network containing two nonlinear phases obeying (33). Results, shown in Fig. (15), are compared to the analytical bound (35). Making use of this formula and of the results presented in Section 2.3 , we also plot the estimate $\xi \approx 1-1.6\left(1-J_{1}\right)^{4 / 3} f^{2 / 3}$ (dotted line). The bound (35) appears meaningful as it displays the same concavity as the FFT data, although FFT results are quite sensitive to the size of the elementary cell $\Omega$.

\section{Conclusion}

The present work examines how one may characterize the effective behavior of a binary mixture made of an insulating phase embedded in a conducting nonlinear phase 


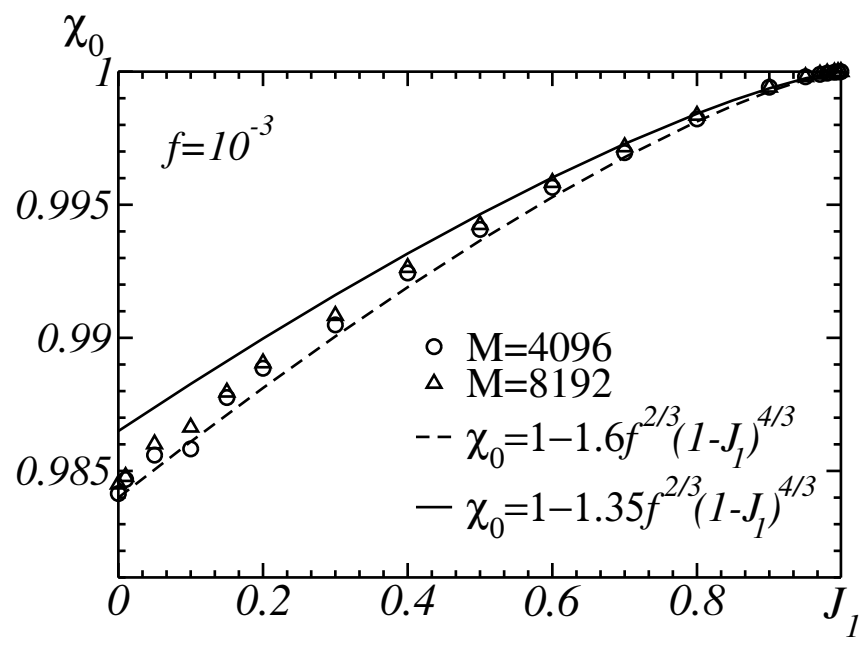

Figure 15: Effective conductivity $\chi_{0}$ of a binary resistor network with yield currents $J_{0}=1$ and $0 \leq J_{1} \leq 1$, as a function of $J_{1}$. Black solid line: bound (35). Dotted line: estimate $\chi_{0} \approx 1-1.6(1-$ $\left.J_{1}\right)^{4 / 3} f^{2 / 3}$ (see text).

characterized by a yield current. The problem is addressed in the context of a random resistor network in two dimensions. We observe that the network's effective conductivity is given by the length of the minimal path defined on the dual lattice. The path spans the network in the direction transverse to the applied field and its length is weighted by the yield current along each bond.

For a dilute concentration of insulating phase, the effective conductivity exhibits singular power-law behavior when the applied macroscopic field is oriented transverse to the directions of the lattice, or if local coupling, that mimic an isotropic law, is introduced between the nodes and the applied field is parallel to the lattice directions. In these two cases, the responses are characterized by a correction $\sim f^{\nu}$ with exponent $\nu$ equal to $1 / 2$ and $2 / 3$ respectively. Furthermore, we derive an upper-bound for the length of geodesics spanning a composite in the continuum, a Boolean set of disks. This bound exhibits an exponent $2 / 3$ in the dilute limit, consistently with numerical data. These results suggest a leading-order correction $\sim f^{2 / 3}$ for the effective conductivity of nonlinear composite materials containing a dilute concentration $f$ of homogeneously-distributed, monodisperse, insulating particles. Other problems of interest include hierarchical microgeometries, some of which are exactly solvable (Idiart and Ponte Castañeda, 2013). As noted by Willot (2015), the present approach may be extended to multiscale structures in the continuum, such as Cox-Boolean random structures. This is currently being investigated.

The present results hold in conductivity as well as in antiplane shear, and are very much similar to the predictions of certain nonlinear homogenization theories for rigid, ideally-plastic porous media under plane strain (Ponte Castañeda, 2002). Indeed, these theories predict that the leading-order correction to the effective yield stress scales as 
$\sim f^{2 / 3}$ as well in the dilute limit $f \rightarrow 0$. In the dual problem of an ideally-plastic media reinforced by rigid particles, strong connections have also been reported between geodesics and shear bands (Jeulin et al., 2008), highlighting the links between effective flow stress, geodesics and the localization patterns of the shear bands (Poliakov et al., 1994).

\section{Appendix A. Algorithm for computing shortest paths in a Boolean model of discs}

Consider a domain containing $M$ disks of centers $\boldsymbol{C}^{1}, \ldots, \boldsymbol{C}^{M}$ and diameter $D$ and set $d_{i j}=\max \left(0 ;\left\|\boldsymbol{C}^{i}-\boldsymbol{C}^{j}\right\|-D\right)$. The distances $d_{1}=d\left(\boldsymbol{A}, \boldsymbol{C}^{1}\right), \ldots, d_{M}=d\left(\boldsymbol{A}, \boldsymbol{C}^{M}\right)$ between a given point $\boldsymbol{A}$ and any disc is computed by the following algorithm:

(i) Set $d_{i}:=\max \left(0 ;\left\|\boldsymbol{C}^{i}-\boldsymbol{A}\right\|-D / 2\right)$.

(ii) For all $i, j$, set $d_{i}:=\min \left(d_{i}, d_{j}+d_{i j}\right)$.

(iii) If any value of $d_{i}$ has been changed in step (ii) GOTO (ii), otherwise STOP.

This algorithm is implemented in a vectorial manner. The lists of coordinates of the disk centers and the list of current $d_{i}$ are saved in memory, while the distances $d_{i j}$ are computed on the fly.

\section{References}

Alava, M., Duxbury, P., 1996. Disorder-induced roughening in the three-dimensional Ising model. Physical Review B 54 (21), 14990.

Bouchaud, J., Bouchaud, E., Lapasset, G., Planes, J., 1993. Models of fractal cracks. Physical review letters 71 (14), 2240.

Bovier, A., Fröhlich, J., Glaus, U., 1986. Lines and domain walls in dilute ferromagnets. Physical Review B 34 (9), 6409.

Dantzig, G., Fulkerson, D. R., 2003. On the max flow min cut theorem of networks. Linear inequalities and related systems 38, 225-231.

De Arcangelis, L., Hansen, A., Herrmann, H., Roux, S., 1989. Scaling laws in fracture. Physical Review B 40 (1), 877.

Deijfen, M., 2003. Asymptotic shape in a continuum growth model. Advances in Applied Probability 35 (2), 303-318.

Derrida, B., Vannimenus, J., 1983. Interface energy in random systems. Physical Review B 27 (7), 4401-4411. 
Donev, A., Musolff, C. E., Duxbury, P. M., 2002. Random manifolds in non-linear resistor networks: applications to varistors and superconductors. Journal of Physics A: Mathematical and General 35 (23), L327.

Drucker, D. C., 1966. The continuum theory of plasticity on macroscale and microscale. Journal of Materials 1 (4), 873-910.

Durrett, R., Liggett, T. M., 1981. The shape of the limit set in richardson's growth model. The annals of Probability 9 (2), 186-193.

Duxbury, P. M., McGarrity, E. S., Holm, E. A., 2006. Critical manifolds in non-linear response of complex materials. Mechanics of materials 38 (8), 757-771.

Elias, P., Feinstein, A., Shannon, C., 1956. A note on the maximum flow through a network. Information Theory, IRE Transactions on 2 (4), 117-119.

Eyre, D., Milton, G., 1999. A fast numerical scheme for computing the response of composites using grid refinement. The European Physical Journal Applied Physics $6(1), 41-47$.

Francescato, P., Pastor, J., Riveill-Reydet, B., 2004. Ductile failure of cylindrically porous materials. part i: plane stress problem and experimental results. European journal of mechanics-A/solids 23 (2), 181-190.

Haslinger, R., Joynt, R., 2000. Theory of percolative conduction in polycrystalline hightemperature superconductors. Physical Review B 61 (6), 4206.

Hoffman, C., 2008. Geodesics in first passage percolation. The Annals of Applied Probability 18 (5), 1944-1969.

Howard, C. D., Newman, C. M., 1997. Euclidean models of first-passage percolation. Probability Theory and Related Fields 108 (2), 153-170.

Huse, D. A., Henley, C. L., 1985. Pinning and roughening of domain walls in Ising systems due to random impurities. Physical review letters 54 (25), 2708.

Idiart, M., Willot, F., Pellegrini, Y.-P., Ponte Castañeda, P., 2009. Infinite-contrast periodic composites with strongly nonlinear behavior: effective-medium theory versus full-field simulations. International Journal of Solids and Structures 46 (18), 33653382.

Idiart, M. I., Ponte Castañeda, P., 2013. Estimates for two-phase nonlinear conductors via iterated homogenization. Proceedings of the Royal Society A: Mathematical, Physical and Engineering Sciences 469 (2153), 20120626.

Jeulin, D., Li, W., Ostoja-Starzewski, M., 2008. On the geodesic property of strain field patterns in elastoplastic composites. Proceedings of the Royal Society A: Mathematical, Physical and Engineering Science 464 (2093), 1217-1227. 
Kardar, M., Zhang, Y.-C., 1987. Scaling of directed polymers in random media. Physical review letters 58 (20), 2087.

Kesten, H., 1987. Percolation theory and first-passage percolation. The Annals of Probability 15 (4), 1231-1271.

Lee, S., 1997. The powerlaws of $m$ and $n$ in greedy lattice animals. Stochastic Processes and their Applications 69, 275-287.

Matheron, G., 1972. Random sets theory and its applications to stereology. Journal of Microscopy 95 (1), 15-23.

Matheron, G., 1975. Random sets and integral geometry. Wiley, New-York.

Michel, J.-C., Moulinec, H., Suquet, P., 2001. A computational scheme for linear and non-linear composites with arbitrary phase contrast. International Journal of Numerical Methods in Engineering 52 (1-2), 139-160.

Poliakov, A. N. B., Herrmann, H. J., Podladchikov, Y. Y., Roux, S., 1994. Fractal plastic shear bands. Fractals 2 (4), 567-581.

Ponte Castañeda, P., 2002. Second-order homogenization estimates for nonlinear composites incorporating field fluctuations. ii - applications. Journal of the Mechanics and Physics of Solids 50 (4), 759-782.

Ponte Castañeda, P., Suquet, P., 1997. Nonlinear composites. In: van Der Giessen, E., Wu, T. Y. (Eds.), Advances in applied mechanics. Vol. 34. Elsevier, San Diego, pp. $171-302$.

Roux, S., François, D., 1991. A simple model for ductile fracture of porous materials. Scripta Metallurgica et Materialia 25 (5), 1087-1092.

Roux, S., Hansen, A., 1992. Perfect plasticity in a random medium. Journal de Physique II 2 (5), 1007-1021.

Sab, K., 1994. Evaluation of the effective strength of a plate with random holes. Comptes rendus de l'académie des sciences Série II 319 (5), 491-497.

Serra, J., 1981. The boolean model and random sets. In: Image Modeling. Academic Press, pp. 343-370.

Willot, F., 2015. The power laws of geodesics in some random sets with dilute concentration of inclusions. Lecture Notes in Computer Science 9082, 535-546.

Willot, F., Abdallah, B., Pellegrini, Y.-P., 2014. Fourier-based schemes with modified green operator for computing the electrical response of heterogeneous media with accurate local fields. International Journal for Numerical Methods in Engineering 98 (7), 518-533. 